University of New Hampshire

University of New Hampshire Scholars' Repository

Physics Scholarship

Physics

$6-2014$

\title{
Radiation belt electron acceleration by chorus waves during the 17 March 2013 storm
}

\author{
W. Li \\ University of California - Los Angeles \\ R. M. Thorne \\ University of California - Los Angeles \\ Q. Ma \\ University of California - Los Angeles \\ B. $\mathrm{Ni}$ \\ University of California - Los Angeles \\ J. Bortnik \\ University of California - Los Angeles
}

See next page for additional authors

Follow this and additional works at: https://scholars.unh.edu/physics_facpub

Part of the Physics Commons

\section{Recommended Citation}

$\mathrm{Li}, \mathrm{W}$., et al. (2014), Radiation belt electron acceleration by chorus waves during the 17 March 2013 storm, J. Geophys. Res. Space Physics, 119, 4681-4693, doi:10.1002/2014JA019945.

This Article is brought to you for free and open access by the Physics at University of New Hampshire Scholars' Repository. It has been accepted for inclusion in Physics Scholarship by an authorized administrator of University of New Hampshire Scholars' Repository. For more information, please contact Scholarly.Communication@unh.edu. 


\section{Authors}

W. Li, R. M. Thorne, Q. Ma, B. Ni, J. Bortnik, D. N. Baker, Harlan E. Spence, Geoffrey Reeves, S. G. Kanekal, J. C. Green, C A. Kletzing, W. S. Kurth, G. B. Hospodarsky, J. B. Blake, Joseph F. Fennell, and S.

Claudepierre 


\section{Journal of Geophysical Research: Space Physics}

\section{RESEARCH ARTICLE \\ 10.1002/2014JA019945 \\ Radiation belt electron acceleration by chorus waves during the 17 March 2013 storm}

\section{Special Section:}

New perspectives on Earth's radiation belt regions from the prime mission of the Van Allen Probes

\section{Key Points:}

- Rapid electron acceleration with a radial PSD peak is observed during a storm

- Chorus driven electron acceleration reproduces observed electron evolution

- Local acceleration by chorus waves is a robust and ubiquitous process

Correspondence to:

W. Li,

moonli@atmos.ucla.edu

\section{Citation:}

Li, W., et al. (2014), Radiation belt electron acceleration by chorus waves during the 17 March 2013 storm, J. Geophys. Res. Space Physics, 119, 4681-4693, doi:10.1002/2014JA019945.

Received 6 MAR 2014 Accepted 30 MAY 2014 Accepted article online 6 JUN 2014 Published online 26 JUN 2014 J. F. Fennell ${ }^{8}$, and S. G. Claudepierre ${ }^{8}$ highly relativistic energies (several MeV).
W. $\mathrm{Li}^{1}$, R. M. Thorne ${ }^{1}$, Q. Ma ${ }^{1}$, B. Ni ${ }^{1}$, J. Bortnik ${ }^{1}$, D. N. Baker ${ }^{2}$, H. E. Spence ${ }^{3}$, G. D. Reeves ${ }^{4}$, S. G. Kanekal ${ }^{5}$, J. C. Green ${ }^{6}$, C. A. Kletzing ${ }^{7}$, W. S. Kurth ${ }^{7}$, G. B. Hospodarsky ${ }^{7}$, J. B. Blake ${ }^{8}$,

${ }^{1}$ Department of Atmospheric and Oceanic Sciences, University of California, Los Angeles, Los Angeles, California, USA, ${ }^{2}$ Laboratory for Atmospheric and Space Research, University of Colorado, Boulder, Colorado, USA, ${ }^{3}$ Institute for the Study of Earth, Oceans, and Space, University of New Hampshire, Durham, New Hampshire, USA, ${ }^{4}$ Space Science and Applications Group, Los Alamos National Laboratory, Los Alamos, New Mexico, USA, ${ }^{5}$ NASA Goddard Space Flight Center, Greenbelt, Maryland, USA, ${ }^{6}$ National Geophysical Data Center, National Oceanic and Atmospheric Administration, Boulder, Colorado, USA, ${ }^{7}$ Department of Physics and Astronomy, University of lowa, lowa City, lowa, USA, ${ }^{8}$ The Aerospace Corporation, Los Angeles, California, USA

Abstract Local acceleration driven by whistler-mode chorus waves is fundamentally important for accelerating seed electron populations to highly relativistic energies in the outer radiation belt. In this study, we quantitatively evaluate chorus-driven electron acceleration during the 17 March 2013 storm, when the Van Allen Probes observed very rapid electron acceleration up to several MeV within $\sim 12$ hours. A clear radial peak in electron phase space density (PSD) observed near $L^{*} \sim 4$ indicates that an internal local acceleration process was operating. We construct the global distribution of chorus wave intensity from the low-altitude electron measurements made by multiple Polar Orbiting Environmental Satellites (POES) satellites over a broad region, which is ultimately used to simulate the radiation belt electron dynamics driven by chorus waves. Our simulation results show remarkable agreement in magnitude, timing, energy dependence, and pitch angle distribution with the observed electron PSD near its peak location. However, radial diffusion and other loss processes may be required to explain the differences between the observation and simulation at other locations away from the PSD peak. Our simulation results, together with previous studies, suggest that local acceleration by chorus waves is a robust and ubiquitous process and plays a critical role in accelerating injected seed electrons with convective energies ( 100 keV) to

\section{Introduction}

Outer radiation belt electron fluxes are highly variable during geomagnetic storms due to a competition between various loss and acceleration processes [Reeves et al., 2003; Bortnik and Thorne, 2007; Li et al., 2006; Li et al., 2007; Xiao et al., 2009; Thorne, 2010; Turner et al., 2013]. Energetic plasma sheet electrons transported into the inner magnetosphere during substorms or periods of enhanced convection provide seed electrons, which can be further energized to highly relativistic energies [Horne and Thorne, 2003; Baker and Kanekal, 2008; Thorne, 2010]. The potential candidates for providing this further acceleration include local acceleration by wave-particle interactions [Summers et al., 2002; Horne et al., 2005a] and inward radial diffusion [Perry et al., 2005; Ukhorskiy et al., 2009]. Specifically, whistler-mode chorus waves are known to provide local acceleration of energetic electrons through efficient energy diffusion [Horne and Thorne, 1998; Summers et al., 2002]. Peaks observed in the electron phase space density (PSD) [Green and Kivelson, 2004; lles et al., 2006; Chen et al., 2007; Reeves et al., 2013] in the heart of the outer radiation belt suggest that an internal local acceleration process is operating. Thorne et al. [2013] simulated the evolution of the electron PSD during the 9 October 2012 storm and found that local acceleration driven by whistler-mode chorus waves was able to reproduce the observed electron PSD increases near the PSD peak location. Their simulation results were not only able to reproduce the magnitude, timing, and energy dependence of the electron PSD, but also the evolution of the electron pitch angle distribution, which provides direct evidence of the rapid electron PSD increase driven by local acceleration of chorus waves during that particular storm. However, whether chorus-driven electron acceleration is always, frequently, or only sometimes, the dominant process responsible for the observed electron energization is a question that needs further investigation.

In the process of simulating radiation belt electron dynamics during a selected event, it is critical to adopt realistic global wave distributions and plasma parameters. However, in situ observations of waves and plasma 
parameters are confined to a limited range in the $L$-shell and magnetic local time (MLT) during each event when observed from equatorial satellites alone. Therefore, statistical global distributions of chorus waves dependent on solar wind parameters and/or geomagnetic indices were used previously to simulate the evolution of electron PSD [e.g., Horne et al., 2005a, 2005b; Albert et al., 2009]. However, a statistical global wave model does not necessarily provide an accurate spatiotemporal evolution of wave intensities in each individual event. More recently, by taking advantage of the extensive coverage of multiple low-altitude satellites, Li et al. [2013] inferred the global distribution of chorus wave intensity from the ratio of the precipitated and trapped electron fluxes measured by Polar Orbiting Environmental Satellites (POES) using a physics-based inversion technique [Ni et al., 2014]. Thorne et al. [2013] adopted the global chorus wave distribution obtained from this technique to simulate the evolution of the electron PSD and showed excellent agreement with the observation during the 9 October 2012 storm, as discussed above. Tu et al. [2014] also simulated this storm with the DREAM3D code using an event-specific global chorus wave model obtained from the POES electron precipitation [Chen et al., 2014] and found that event-specific global chorus wave model and realistic seed electron population play an important role in reproducing the observed electron dynamics.

In the present study, we apply the technique shown in Li et al. [2013] to construct the global distribution of chorus wave intensity, which is used to simulate the electron PSD evolution during another interesting geomagnetic storm, which occurred on 17 March 2013 and caused rapid electron acceleration up to several $\mathrm{MeV}$ within $\sim 12 \mathrm{~h}$. Relativistic electron dynamics and chorus wave activity during this storm have been analyzed in Baker et al. [2014] and Foster et al. [2014] using in situ Van Allen Probes observations. They found that substorm processes play a significant role in creating a seed electron population and chorus wave enhancements are associated with a fast energization of relativistic electrons. In this study, by solving the 2-D Fokker-Planck equation, we quantitatively examine whether chorus-driven acceleration can lead to the observed electron PSD, in terms of its evolution in magnitude, timing, and pitch angle distributions during this geomagnetic storm at various locations, not only near the peak in PSD but also in the regions away from the peak.

\section{Data Analysis}

\subsection{Van Allen Probes Data Analysis}

Particle and wave measurements from the twin Van Allen Probes near the equatorial magnetosphere [Mauk et al., 2012] were used to analyze the 17 March 2013 geomagnetic storm. Energetic electron measurements, which are used to analyze the seed electron population and relativistic radiation belt electrons and their pitch angle distributions, are obtained from both the Relativistic Electron Proton Telescope (REPT) [Baker et al., 2012] and the Magnetic Electron lon Spectrometer (MagEIS) instruments [Blake et al., 2013], which are elements of the Energetic Particle, Composition, and Thermal Plasma instrument suite [Spence et al., 2013]. The Electric and Magnetic Field Instrument Suite and Integrated Science (EMFISIS) provides measurements of DC magnetic fields (magnetometer instrument) and a comprehensive set of wave electric and magnetic fields (Waves instrument) [Kletzing et al., 2013]. The Waveform Receiver (WFR) on the EMFISIS Waves instrument measures wave power spectral density from $10 \mathrm{~Hz}$ up to $12 \mathrm{kHz}$ [Kletzing et al., 2013], which is well suited to measure whistler-mode chorus waves. The High Frequency Receiver (HFR) is designed to provide electric spectral intensity between 10 and $400 \mathrm{kHz}$, thus enabling measurements of the upper hybrid resonance frequency, from which the total plasma density can be calculated. The upper hybrid resonance frequency or the lower frequency cutoff of the continuum radiation (which provides the upper limit of the plasma density) is used to identify the location of the plasmapause and to determine the plasma density in the outer radiation belt used in our simulation.

\subsection{Construction of the Global Chorus Wave Intensities From the POES Electron Measurements}

Chorus wave amplitudes are inferred from the ratio of the precipitated and trapped electron fluxes over the energy range of 30-100 keV measured by multiple POES satellites. Based on the fact that lower-band chorus waves play a dominant role in driving 30-100 keV electron precipitation [e.g., Lam et al., 2010; Thorne et al., 2010], using quasi-linear diffusion theory [Kennel and Petschek, 1966], we developed a physics-based technique to link the electron pitch angle distribution near the bounce loss cone driven by chorus wave scattering and the twodirectional electron measurements by POES satellites over the energy range of 30-100 keV [Evans and Greer, 2004; 
Green, 2013]. Details of this technique are described in Li et al. [2013] and Ni et al. [2014]. Since multiple POES satellites are present over a wide range of MLT with an orbital period of $\sim 100 \mathrm{~min}$, inferred chorus wave amplitudes from the ratio of precipitated and trapped electron fluxes provide extensive coverage in $L$ and MLT with a time resolution comparable to $\sim 0.5-1 \mathrm{~h}$. This technique has been validated by analyzing a number of conjunction events between the Van Allen Probes and POES satellites and is confirmed to provide reasonable estimates on the whistler-mode wave amplitudes including both chorus and hiss [Li et al., 2013, 2014]. This data-driven dynamic global chorus distribution is used in our simulation to quantify chorus-driven electron acceleration in the outer radiation belt, as discussed in section 3.2 .

\section{Observational and Simulation Results}

\subsection{Observation of Electrons and Chorus Waves}

An overview of the 17 March 2013 storm is shown in Figure 1, which includes solar wind parameters, geomagnetic indices, and electron fluxes observed by both MagEIS and REPT. Over the period between 06 and 09 UT on 17 March 2013, the solar wind dynamic pressure (Figure 1a) suddenly increased associated with the shock arrival [Baker et al., 2014], which pushed the magnetopause location down to $\sim 6 R_{\mathrm{E}}$ at noon according to the predicted magnetopause location by Shue et al. [1998], as shown in Figure 1c. In association with this compression of the magnetopause, relativistic electron fluxes ( $>\sim 2 \mathrm{MeV}$ ) decreased by almost an order of magnitude from Trajectory 2 to 3 (see Figure $1 \mathrm{j}$ ), which is likely due to the magnetopause shadowing effect and the following outward radial diffusion process [e.g., Shprits et al., 2006; Turner et al., 2012]. Subsequently, electron fluxes with energies below a few MeV exhibited rapid increases from Trajectory 3 to 4 within several hours, while higher-energy electron fluxes above $\sim 3 \mathrm{MeV}$ gradually increased during the following $\sim 10 \mathrm{~h}$ until Trajectory 6 , after which electron fluxes exhibited insignificant changes. During this acceleration interval from Trajectory 3 to 6 (red horizontal line shown in Figure 1), the interplanetary magnetic field (IMF) $B_{z}$ was mostly southward (Figure 1b), the predicted magnetopause location generally stayed above $\sim 8 R_{\mathrm{E}}$ (Figure 1c), and strongly negative values were recorded in both SYM-H (Figure 1d) and AL (Figure 1e), all of which favor electron acceleration by enhancing the seed electron fluxes and various wave activities and reducing the magnetopause loss [e.g., Kataoka and Miyoshi, 2008; McPherron et al., 2009; Thorne et al., 2013]. We simulate the electron PSD evolution during this acceleration interval from $\sim 12$ to 24 UT on 17 March 2013, which is described in section 3.2 below.

We calculated the evolution of the electron PSD for a constant first adiabatic invariant $(\mu=3433 \mathrm{MeV} / \mathrm{G})$ and second adiabatic invariant $\left(K=0.10 \mathrm{G}^{1 / 2} R_{\mathrm{E}}\right)$ along the trajectories of both Van Allen Probes $\mathrm{A}$ and $\mathrm{B}$ during a more extended time interval from 09:07 UT on 17 March to 08:32 UT on 18 March to show a more complete sequence of the PSD evolution around the acceleration interval. The calculated electron PSD is shown in Figure 2 as a function of $L^{*}$, color-coded for different times, and is generally consistent with the PSD values shown in Baker et al. [2014]. Note that $L^{*}$ is calculated for $90^{\circ}$ pitch angle particles using the TS05 model [Tsyganenko and Sitnov, 2005]. For this given $\mu=3433 \mathrm{MeV} / \mathrm{G}$, the corresponding electron energy varies from $\sim 2-3 \mathrm{MeV}$ at $L^{*}=5$ to $\sim 6 \mathrm{MeV}$ at $L^{*}=3$. For the provided $K=0.10 \mathrm{G}^{1 / 2} R_{\mathrm{E}}$, the corresponding electron pitch angles range from $\sim 40^{\circ}$ to $\sim 90^{\circ}$ during the trajectories of Van Allen Probes $A$ and $B$, which were mostly within $15^{\circ}$ of the magnetic equator over the $L^{*}$ between 3 and 5. Before $\sim 12$ UT on 17 March, the PSD was very low $\left(<\sim 10^{-10}\right.$ $\left.\left(\mathrm{c} \mathrm{MeV}^{-1} \mathrm{~cm}^{-1}\right)^{3}\right)$, probably due to the substantial loss caused by the magnetopause shadowing effect associated with the relatively large dynamic pressure increases over 06-11 UT on 17 March. The electron PSD started to increase from Pass 2 and continued to increase until Pass 7 and 8, where a clear peak in PSD was observed at $L^{*}$ of 4.1-4.3. Subsequently, the peak was smoothed out predominantly toward larger $L^{*}$, leading to a decreased PSD at $L^{*}$ between 4.1 and 4.3 and an increased PSD at $L^{*}>4.7$. This rising peak in PSD from Pass 2 to 8 is likely to be caused by an internal acceleration process, while the flattened PSD profile is probably due to outward radial diffusion [e.g., Shprits et al., 2006; Turner et al., 2012]. In Figure 2, the PSD values were not shown if the corresponding electron energy for the fixed $\mu$ was less than $2 \mathrm{MeV}$ (the lower limit of REPT energy channel) or larger than $~ 5.6 \mathrm{MeV}$, above which electron fluxes are close to the background level, or $L^{*}$ from TS05 was not defined because it fell on an open drift boundary. Here we focus on simulating the electron PSD evolution during the acceleration time interval from Pass 2 to 8, approximately from 12 to 24 UT on 17 March 2013.

Since we evaluate electron acceleration driven by chorus waves, in Figure 3, we show the chorus waves directly observed by the EMFISIS instrument and inferred from the POES electron measurements using the 


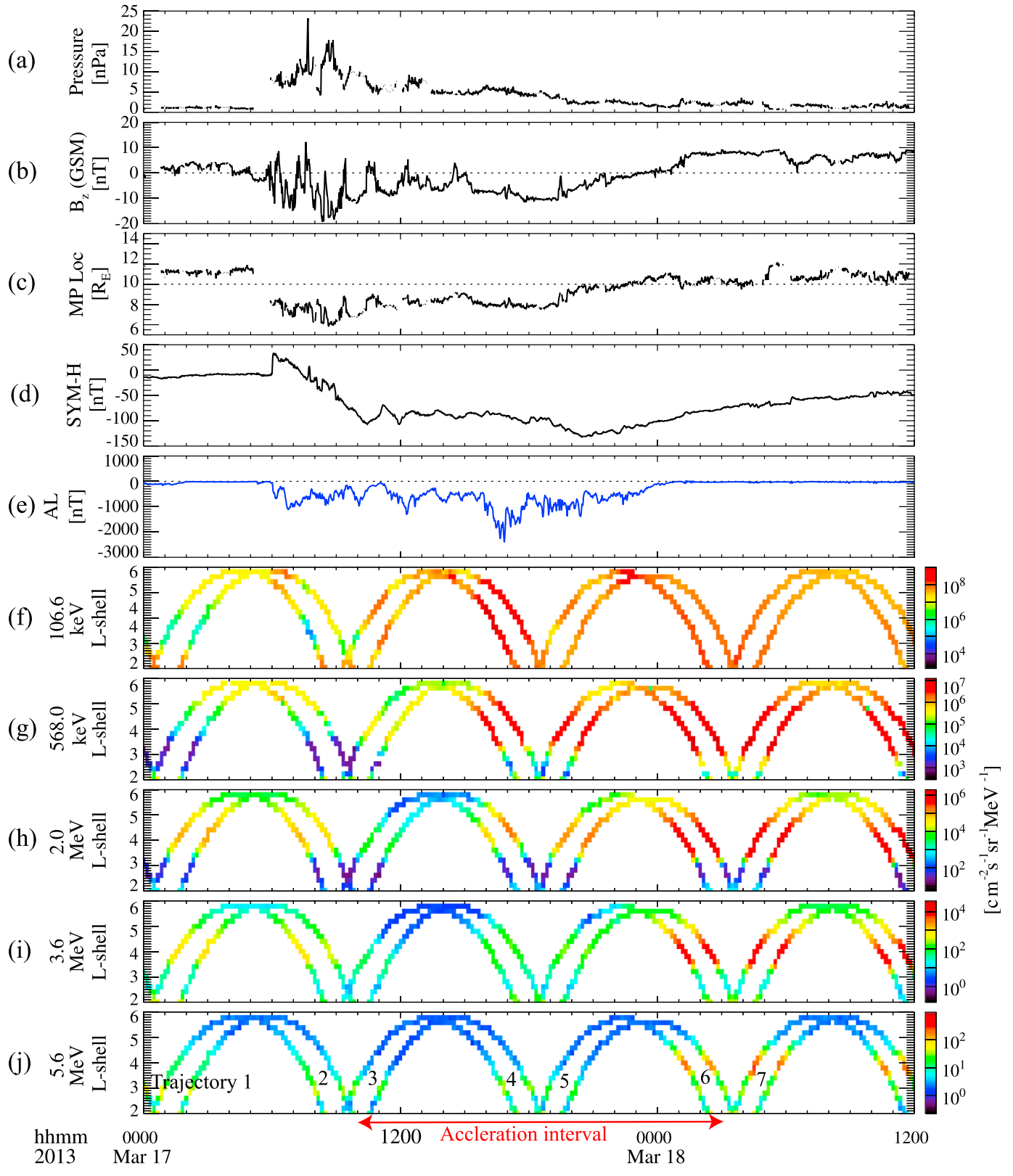

Figure 1. Solar wind parameters, geomagnetic indices, and energetic electron fluxes observed by the Van Allen Probes during the 17 March 2013 storm. (a) Solar wind dynamic pressure, (b) the $z$ component of the interplanetary magnetic field in the GSM coordinate, (c) The magnetopause location obtained using the Shue et al. [1998] model, (d) SYM-H, and (e) AL index. (f)-(j) Electron fluxes observed by MagEIS and REPT on both Van Allen Probes A and B at various energies. The red horizontal arrow represents the acceleration time interval, during which our simulation is performed.

inversion technique described above. Since the apogee of the Van Allen Probes was located near the midnight sector during this storm, the outbound (inbound) pass corresponds to the premidnight (postmidnight) sector. The EMFISIS wave observations (Figure $3 \mathrm{~b}$ and $3 \mathrm{c}$ ) show that chorus waves remained strong during the acceleration interval associated with strong disturbances in the AL index (Figure 3a) near the nightside. Note that during this interval, the plasmapause was compressed to below $3 R_{\mathrm{E}}$, which can be inferred from the upper hybrid resonance line detected from the EMFISIS instrument [Foster et al., 2014]. Chorus wave amplitudes inferred from the two-directional POES electron measurements are shown with a bin size of $1 \mathrm{~h} \times 0.2 L$ over six different MLT ranges (Figures $3 \mathrm{~d}-3 \mathrm{i}$ ). The comparison of directly measured and 


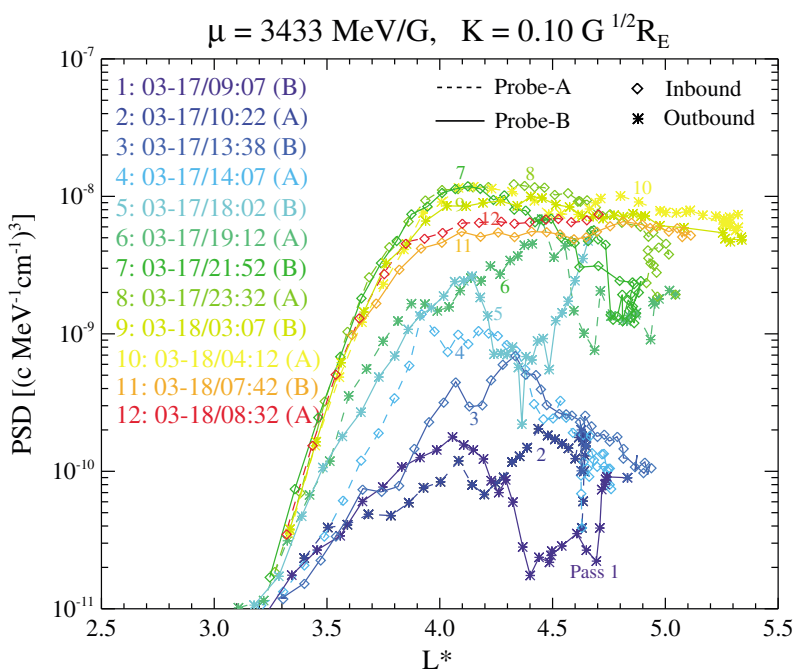

Figure 2. The time evolution (color-coded) of electron PSD as a function of $L^{*}$ (TS05) for $\mu=3433 \mathrm{MeV} / \mathrm{G}$ and $K=0.10 \mathrm{G}^{1 / 2} R_{\mathrm{E}}$ from $\sim 09: 07$ UT on 17 March to $~ 08: 32$ UT on 18 March in 2013 observed by both Van Allen Probes A and B. The time indicates the start of either inbound $(\circ)$ or outbound (asterisk) and dashed (solid) line represents the trajectory of Van Allen Probe A (B). and postmidnight (00-04 MLT) sector, respectively. In other MLT sectors, we scaled the density values by a factor of 0.43 from the statistical plasmatrough density model [Sheeley et al., 2001], since 0.43 is the ratio between the measured electron density and the statistical model value at $L \sim 4.25$ over 00-04 MLT. The ambient plasma density is further assumed to be constant as a function of latitude and remain constant over the $12 \mathrm{~h}$ acceleration interval.

To evaluate the quasi-linear diffusion coefficients of radiation belt electrons driven by whistler-mode chorus, we assume that the chorus wave power spectral density $I_{B}(f)$ follows a Gaussian frequency distribution with lower and upper cutoffs $f_{l c}$ and $f_{u c}$, the median value $f_{m}$, and the bandwidth $\delta f$ :

$$
I_{B}(f)=\frac{2\left|B_{w}\right|^{2}}{\pi^{1 / 2} \delta f}\left[\operatorname{erf}\left(\frac{f_{m}-f_{l c}}{\delta f}\right)+\operatorname{erf}\left(\frac{f_{u c}-f_{m}}{\delta f}\right)\right]^{-1} \exp \left[-\left(\frac{f-f_{m}}{\delta f}\right)^{2}\right],
$$

where $\left|B_{w}\right|^{2}$ is the averaged chorus magnetic wave power and erf is the error function. We set $f_{l c} / f_{\text {ce }}=0.05$ and $f_{u d} / f_{\text {ce }}=0.5$, while $f_{m} / f_{\text {ce }}$ and $\delta f / f_{\text {ce }}$ change with the MLT sector, as shown in Table 1 . The wave normal distribution of chorus is also assumed to be Gaussian and given by

$$
g(\theta)=\exp \left[-\left(\frac{\tan \theta-\tan \theta_{m}}{\tan \theta_{w}}\right)^{2}\right] \quad\left(\theta_{l c} \leq \theta \leq \theta_{u c}\right),
$$

where $\theta$ is the wave normal angle, $\theta_{m}$ the peak, $\theta_{w}$ the angular width, and $\theta_{l c}$ and $\theta_{u c}$ the lower and upper bounds to the wave normal distribution, outside which the wave power is zero. Here we use a frequently adopted model for the chorus wave normal angle distribution, that is, $\theta_{l c}=0^{\circ}, \theta_{u c}=45^{\circ}, \theta_{m}=0^{\circ}$, and $\theta_{w}=30^{\circ}$ [e.g., Horne et al., 2005b; Thorne et al., 2013], to represent the quasi-parallel lower-band chorus waves particularly during active times [e.g., Li et al., 2011; Agapitov et al., 2013; Artemyev et al., 2013]. The latitudinal extent $(|\lambda|)$ of chorus waves varies in different MLT sectors based on the statistical results [Meredith et al., 2012; Agapitov et al., 2013].

\subsection{Simulation of Energetic Electron Acceleration}

Drift and bounce averaged diffusion coefficients driven by lower-band chorus waves at $L=4.25$ are computed using the UCLA full diffusion code [Ni et al., 2008, 2011; Shprits and Ni, 2009] in a dipole magnetic field model based on the parameters shown in Table 1. Note that to simulate the stretching of the nightside magnetic field associated with the strong storm-time geomagnetic activity, we adopted the dipole field 


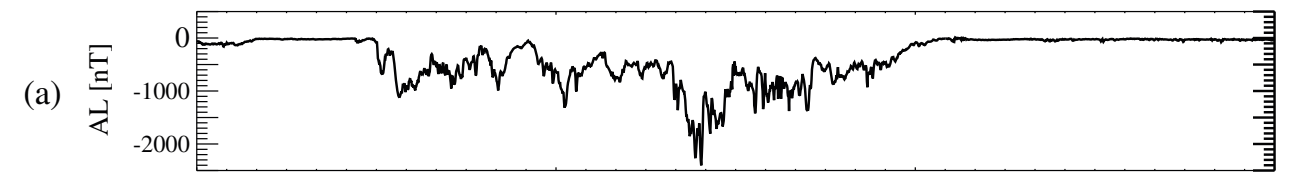

(b)

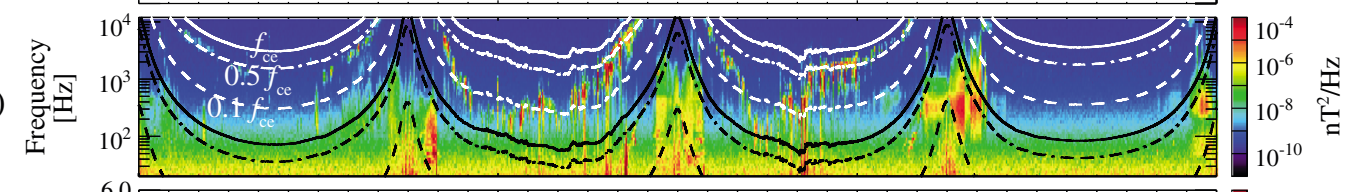

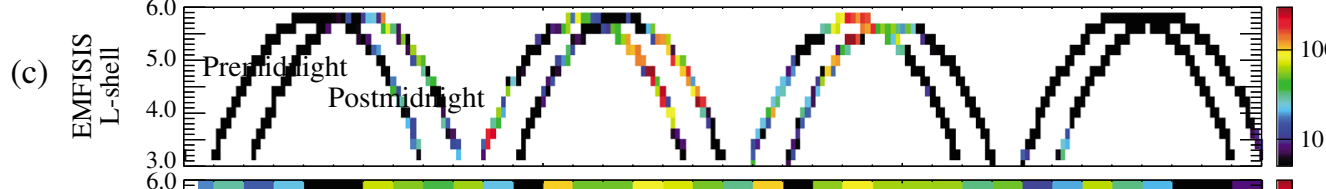
$\frac{\text { F }}{3}$

(d)

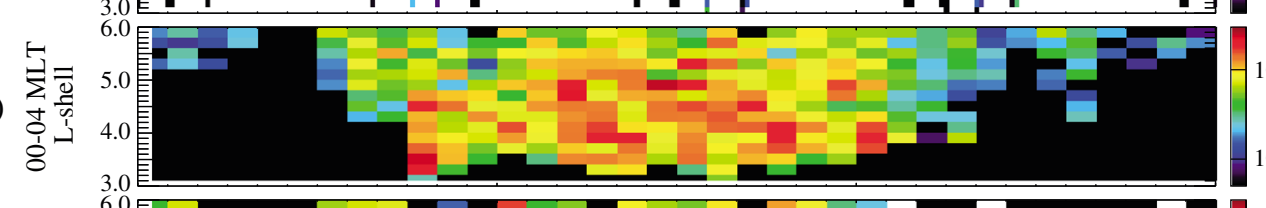

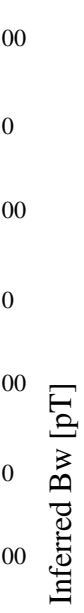

(e)

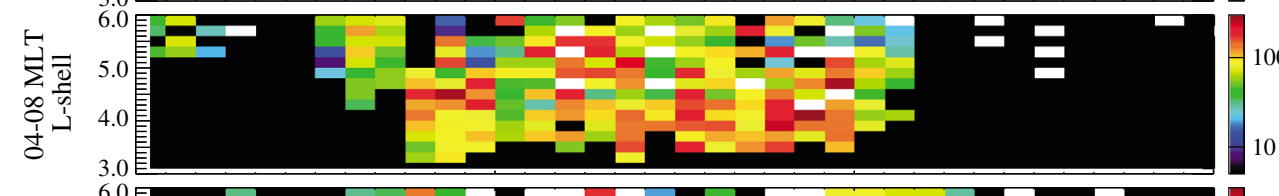

(f)
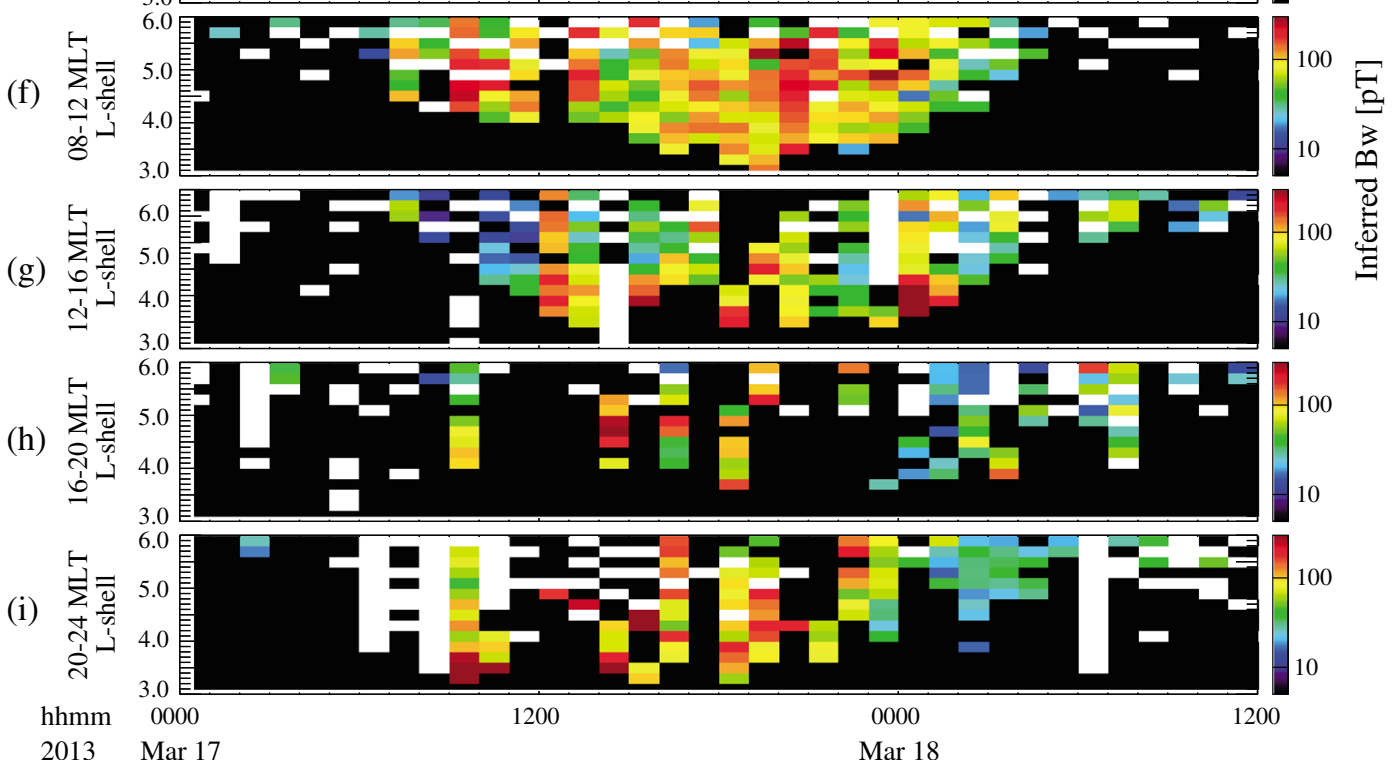

Figure 3. The evolution of the chorus wave distribution during the 17 March storm. (a) AL index, and (b) frequency-time spectrogram of magnetic field spectral density observed by Van Allen Probe B along its trajectory. White lines in

Figure $3 \mathrm{~b}$ indicate $f_{\text {ce }}$ (solid), $0.5 f_{\text {ce }}$ (dash-dotted), and $0.1 f_{\text {ce }}$ (dashed), and black lines represent $f_{\text {LHR }}$ (solid) and $0.5 f_{\text {LHR }}$ (dash-dotted), where $f_{\text {ce }}$ and $f_{\text {LHR }}$ are the electron cyclotron frequency and lower hybrid resonance frequency, respectively (c) Lower-band chorus wave amplitudes integrated over 0.05- $0.5 f_{\text {ce }}$ as a function of $L$-shell observed by both Van Allen Probes, where the outbound (inbound) corresponds to the premidnight (postmidnight) sector. (d)-(i) Global distribution of chorus wave amplitudes inferred from the 30-100 keV electron population measured by multiple Polar Orbiting Environmental Satellites (POES) satellites over various magnetic local time (MLT) ranges.

configuration but scaled the background magnetic field intensity by a factor of 0.69 for the 00-04 MLT and 20-24 MLT sectors according to the in situ magnetic field intensity measured by Van Allen Probes. We calculated the diffusion coefficients including cyclotron harmonic resonances from -10 to 10 and the Landau resonance. The net scattering rates of pitch angle $\left(\left\langle D_{\alpha_{e q} a_{e q}}\right\rangle\right)$, momentum $\left(\left\langle D_{p p}\right\rangle\right)$, and mixed diffusion coefficients $\left(\left\langle D_{\alpha_{e q p}}\right\rangle\right)$ for energetic electrons due to the interaction with chorus waves at $L=4.25$ are shown as a function of equatorial pitch angle $\left(\alpha_{\mathrm{eq}}\right)$ and kinetic energy $(E)$ in Figure 4 over three time intervals of 12-16, 
Table 1. Adopted Model Parameters at $L=4.25$ in Various Magnetic Local Time (MLT) Ranges, Showing Chorus Wave Spectrum, Wave Latitudinal Extension $(|\lambda|)$, Plasma Density $\left(N_{\mathrm{e}}\right)$, the Ratio of Plasma to Electron Cyclotron Frequency $\left(f_{\mathrm{pe}} / f_{\mathrm{ce}}\right)$, and Chorus Wave Amplitudes $\left(B_{\mathrm{w}}\right)$

\begin{tabular}{|c|c|c|c|c|c|}
\hline & $f_{m} / f_{\mathrm{ce}}$ & $\delta f / f_{\mathrm{ce}}$ & $|\lambda|$ & $N_{\mathrm{e}}\left(\mathrm{cm}^{-3}\right)$ & $f_{\mathrm{pe}} / f_{\mathrm{ce}}$ \\
\hline 00-04 MLT & 0.25 & 0.1 & $<10^{\circ}$ & 9.3 & 3.5 \\
\hline 04-08 MLT & 0.23 & 0.1 & $<15^{\circ}$ & 9.5 & 2.4 \\
\hline 08-12 MLT & 0.21 & 0.08 & $<45^{\circ}$ & 13.5 & 2.9 \\
\hline 12-16 MLT & 0.2 & 0.08 & $<40^{\circ}$ & 17.4 & 3.3 \\
\hline 16-20 MLT & 0.2 & 0.06 & $<25^{\circ}$ & 17.2 & 3.3 \\
\hline 20-24 MLT & 0.22 & 0.08 & $<10^{\circ}$ & 13.1 & 4.2 \\
\hline \multicolumn{6}{|c|}{$B_{\mathrm{w}}(\mathrm{pT})$} \\
\hline & Mar. 17 & \multicolumn{2}{|c|}{ Mar. 17} & \multicolumn{2}{|c|}{ Mar. 17} \\
\hline & $12-16$ UT & \multicolumn{2}{|c|}{ 16-20 UT } & \multicolumn{2}{|c|}{ 20-24 UT } \\
\hline 00-04 MLT & 115.7 & \multicolumn{2}{|c|}{131.9} & \multicolumn{2}{|c|}{117.5} \\
\hline 04-08 MLT & 100.2 & \multicolumn{2}{|c|}{120.9} & \multicolumn{2}{|c|}{144.0} \\
\hline 08-12 MLT & 68.7 & \multicolumn{2}{|c|}{118.4} & \multicolumn{2}{|c|}{110.4} \\
\hline $12-16 \mathrm{MLT}$ & 112.8 & \multicolumn{2}{|c|}{79.3} & \multicolumn{2}{|c|}{118.8} \\
\hline 16-20 MLT & 52.7 & \multicolumn{2}{|c|}{44.4} & \multicolumn{2}{|c|}{10.9} \\
\hline 20-24 MLT & 140.8 & \multicolumn{2}{|c|}{107.1} & \multicolumn{2}{|c|}{57.4} \\
\hline
\end{tabular}

16-20, and 20-24 UT. Both pitch angle and momentum diffusion coefficients are stronger at large pitch angles $\left(>40^{\circ}\right)$ at energies above hundreds of keV. However, at small pitch angles, the pitch angle diffusion significantly dominates over the momentum diffusion in a broad range of electron energies. During these three time intervals, all of the three diffusion coefficients did not exhibit significant changes, which is consistent with the similar averaged wave activities during the period of 12-24 UT on 17 March, as shown in Figure 3.

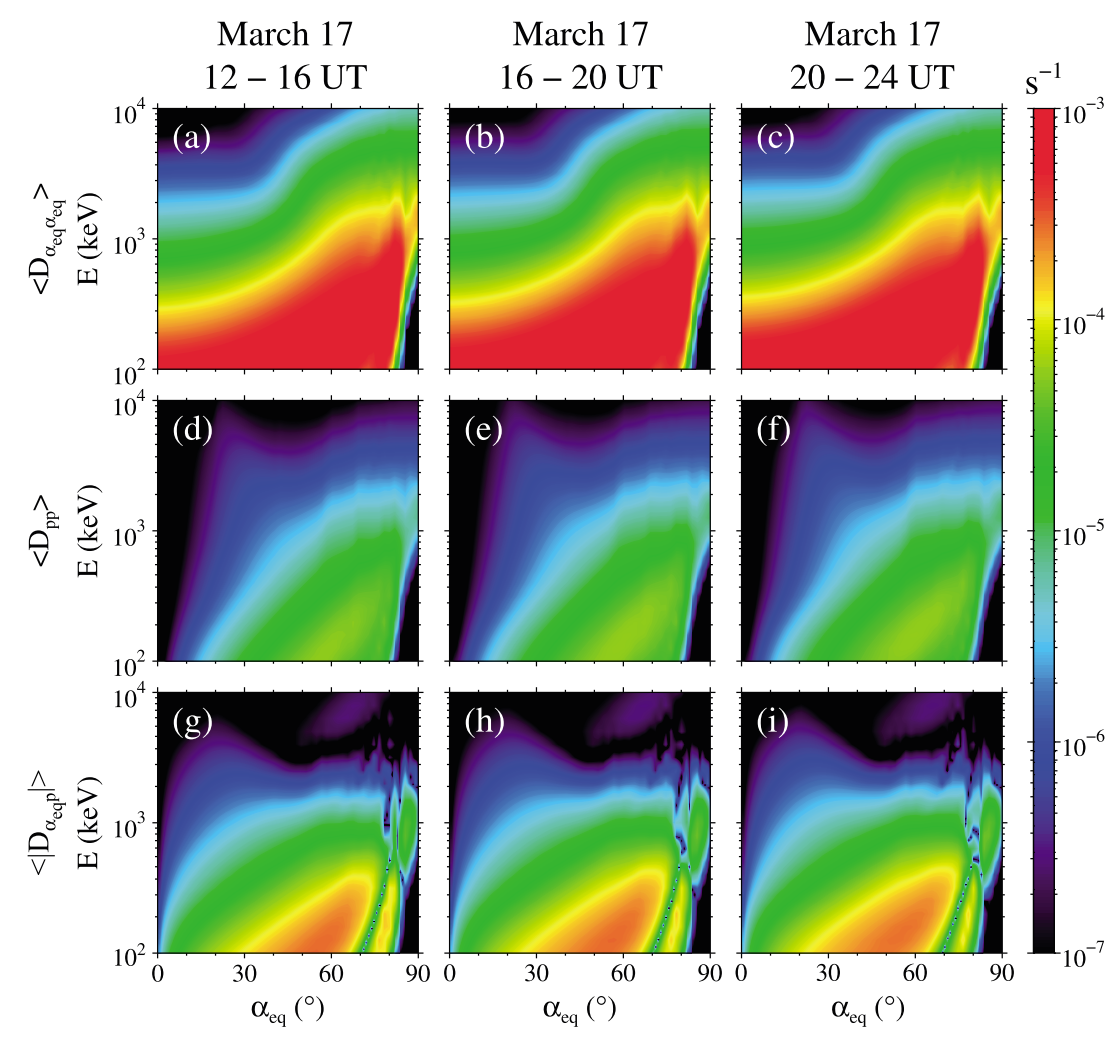

Figure 4. (a)-(c) Bounce and drift averaged pitch angle diffusion coefficients as a function of equatorial pitch angle $\left(\alpha_{\text {eq }}\right)$ and electron energy $(E)$ during the intervals 12-16, 16-20, and 20-24 UT of 17 March 2013. (d)-(f) The same as Figures 4a-4c but for momentum diffusion coefficients. (g)-(i) The same as Figures $4 \mathrm{a}-4 \mathrm{c}$ but for mixed diffusion coefficients. 

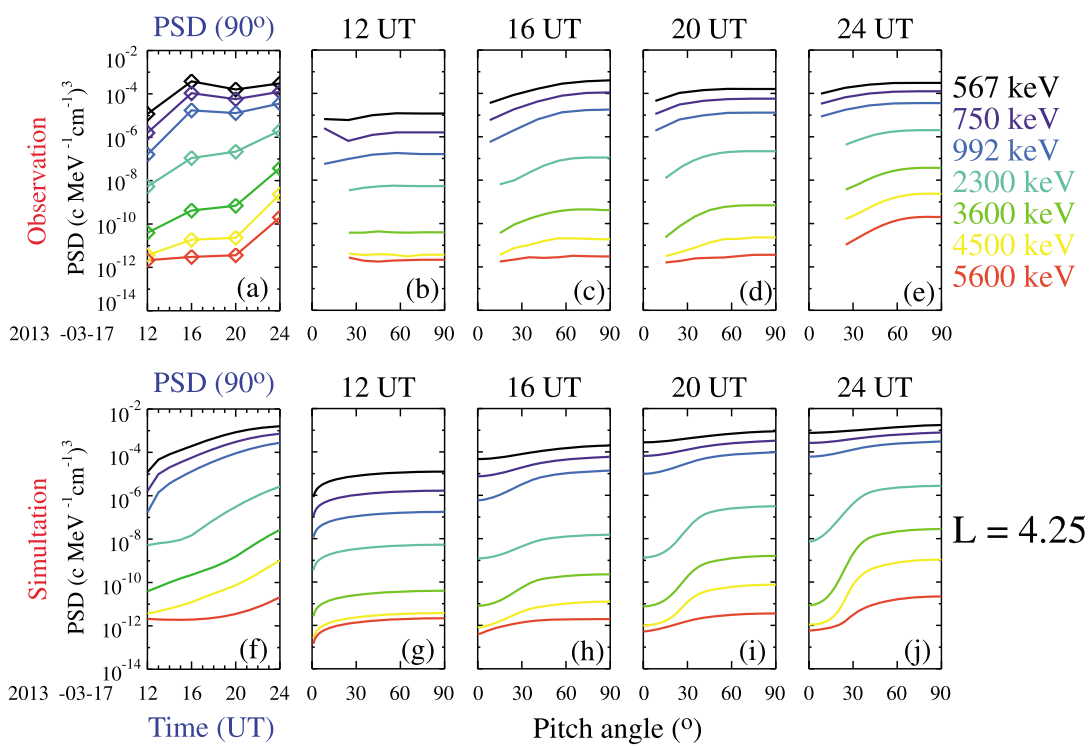

Figure 5. Comparison of observational and simulation results of the electron PSD evolution at energies from $567 \mathrm{keV}$ to $5600 \mathrm{keV}$ at $L \sim 4.25$. (a) Increases of perpendicular electron PSD observed by both MagEIS and REPT during 12-24 UT on 17 March. (f) The Fokker-Planck simulation results of perpendicular electron PSD with the same format as Figure 5a. (b)-(e) The observed evolution of the electron pitch angle distribution with $4 \mathrm{~h}$ time intervals from 12 to 24 UT on 17 March. (g)-(j) Fokker-Planck simulation results of the electron pitch angle evolution with the same format as Figures 5b-5e.

In order to simulate the electron PSD evolution, we solved the 2-D Fokker-Planck equation over the energy range $0.1-30 \mathrm{MeV}$, similar to the study by Thorne et al. [2013]. The initial electron energy spectrum at $90^{\circ}$ equatorial pitch angle was adopted from a spline fitting of the electron PSD as a function of energy (not shown) between $\sim 100 \mathrm{keV}$ and $8.8 \mathrm{MeV}$ measured by MagEIS and REPT on both Van Allen Probes during Trajectory 3 (Figure 1) just before 12 UT on $17 \mathrm{March}$, and was extrapolated from 8.8 to $30 \mathrm{MeV}$ by assuming a power law distribution at higher energies. The initial pitch angle distribution of the electron PSD $(f)$ is assumed to be $f\left(\alpha_{\mathrm{eq}}, p\right)=f\left(\alpha_{\mathrm{eq}}=90^{\circ}, p\right)\left(\sin \alpha_{\mathrm{eq}}\right)^{n}$, where $n=0.65$, which was obtained by fitting the pitch angle distribution of the observed electron PSD at $\sim 100 \mathrm{keV}$. The electron PSD at the lower energy boundary (100 keV) was kept at a constant value, which was obtained by averaging over this $12 \mathrm{~h}$ acceleration time interval, and the electron PSD at the upper energy boundary $(30 \mathrm{MeV})$ was set to a constant value. The lower and upper boundary conditions for the equatorial pitch angle distribution were set as $\partial f\left(\alpha_{\mathrm{eq}}=0^{\circ}, p\right) / \partial \alpha_{\mathrm{eq}}=0$ and $\partial f\left(\alpha_{\mathrm{eq}}=90^{\circ}, p\right) / \partial \alpha_{\mathrm{eq}}=0$. The 2-D Fokker-Planck equation was numerically solved by using the standard alternative direction implicit method for a time interval of $12 \mathrm{~h}$ with a time step of $1 \mathrm{~s}$. The drift and bounce averaged diffusion coefficients were updated every $4 \mathrm{~h}$ corresponding to the diffusion coefficients shown in Figure 4.

Figure 5 shows the electron PSD evolution over a broad range of energies from $\sim 567$ to $5600 \mathrm{keV}$ at $L=4.25$ near the PSD peak location. Note that the observation result shown at the designated time is obtained by averaging the observed electron PSD from both Van Allen Probes A and B over the L-shell between 4.0 and 4.5 within $\pm 2 \mathrm{~h}$ of the indicated time. The comparison of the observed (Figure $5 \mathrm{a}$ ) and simulated electron PSD evolution at $\sim 90^{\circ}$ pitch angles (Figure 5f) overall indicates excellent agreement with the magnitude, timing, and energy dependence. Lower-energy electrons were accelerated first followed by higher-energy electrons with a time delay of several hours and several-MeV electrons experienced nearly 2 orders of magnitude increases in PSD during this acceleration interval. Note that the seed electron PSD (hundreds of keV) increased by almost two orders of magnitude from $\sim 12$ to $\sim 16$ UT associated with the substantial decreases in the AL index (Figure 1e) caused by strong substorm activity, followed by a decrease in PSD by nearly an order of magnitude from $\sim 16$ to $\sim 20$ UT corresponding to the reduced substorm activity. However, these in situ seed electron populations were observed near the midnight sector, where the substorm injection preferentially occurs, and thus the observed seed electron population near the midnight sector may not necessarily represent the electron PSD averaged over all MLT sectors. This variation of the electron PSD at a few hundreds keV led to corresponding PSD changes for electron energies below $\sim 1 \mathrm{MeV}$. Consequently, the simulated evolution of electron PSD was not very consistent with the observation at energies below $\sim 1 \mathrm{MeV}$, 

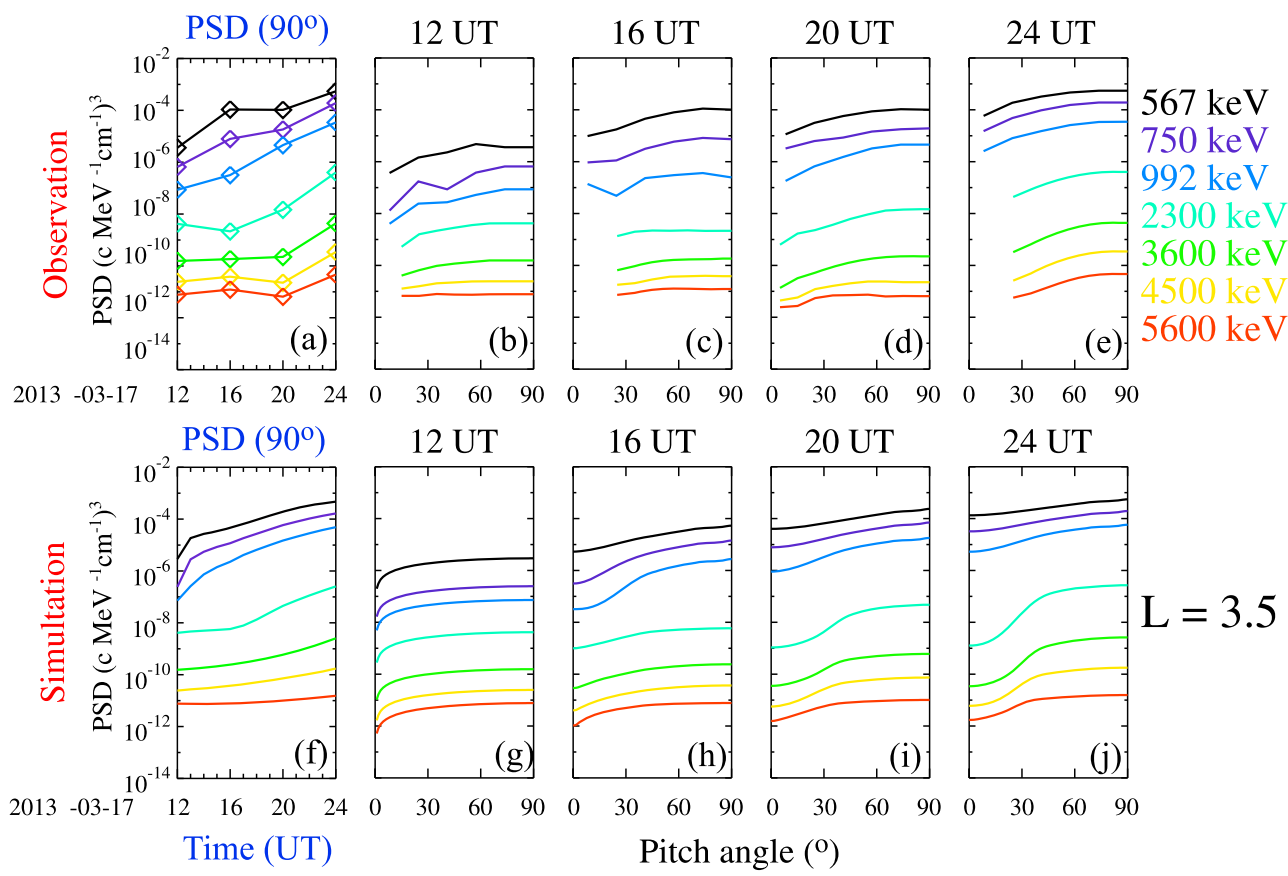

Figure 6. The same as Figure 5 but at $L=3.5$.

partially because we adopted a fixed lower boundary condition (at $100 \mathrm{keV}$ ) averaged over the entire acceleration interval. We also performed the simulation by updating the PSD values at the lower-energy boundary of $100 \mathrm{keV}$ (not shown), and the simulated electron PSD below $\sim 1 \mathrm{MeV}$ was even larger than the simulation results shown in Figure 5 due to the substantial increase in electron PSD at 16 UT but lack of mechanisms of reducing them, such as electron loss due to the open drift trajectory, pitch angle scattering by hiss in plumes, and radial diffusion process. Note that during this storm the plasmapause location was compressed down to $<3 R_{E}$, which suggests the presence of the strong storm time convection electric field, and thus $\sim 100 \mathrm{keV}$ electrons in the location at above $\sim 3.5 R_{\mathrm{E}}$ are likely to be located on open drift orbits and ultimately lost to the magnetopause [e.g., Chen et al., 2003]. However, the several-MeV electron PSD evolution was less affected by this significant seed electron PSD variation, showing a gradual increase with time, and was very similar in both results of using the fixed and floating lower energy boundary. Furthermore, we also performed the simulation until 04 UT on 18 March 2013 and the simulation result is almost identical to Figure 5j, since the chorus wave activity was overall very weak over 00-04 UT on 18 March 2013, as shown in Figure 3.

We also compare the evolution of the electron pitch angle distribution from the observation and simulation in Figures $5 b-5 e$ and Figures $5 \mathrm{~g}-5 \mathrm{j}$. We show the observed and simulated electron pitch angle distributions in separate panels in order to focus on their overall buildup on a timescale of several hours and the evolution of electron pitch angle distributions rather than detailed differences between them. We note that the indicated timing is not very precise due to the averaged PSD values within $\pm 2 \mathrm{~h}$ of the indicated time over the $L$-shell of 4.0-4.5, since electron data from the Van Allen Probes are only available every $\sim 4-5 \mathrm{~h}$ in the same $L$-shell range. Overall, the similarities between the observed and simulated electron pitch angle distributions are quite impressive. First, they both show a flat distribution at pitch angles above $\sim 50^{\circ}$, which is due to the larger electron pitch angle and momentum diffusion coefficients over relatively large pitch angles. Second, the gradient toward the loss cone is smaller at lower energies and larger at higher energies, which is entirely consistent with the profile of pitch angle and momentum diffusion coefficients, that are flat for a wider (narrower) range of pitch angles at lower (higher) energies. Third, the large gradient toward the loss cone shown both in the observation and simulation is direct evidence of the dominance of the momentum diffusion over the pitch angle scattering loss caused by chorus waves. However, this gradient is larger in the simulation compared to the observation, which might be due to the lack of other possible mechanisms of scattering electrons, such as electron scattering by hiss in plumes or radial diffusion. 

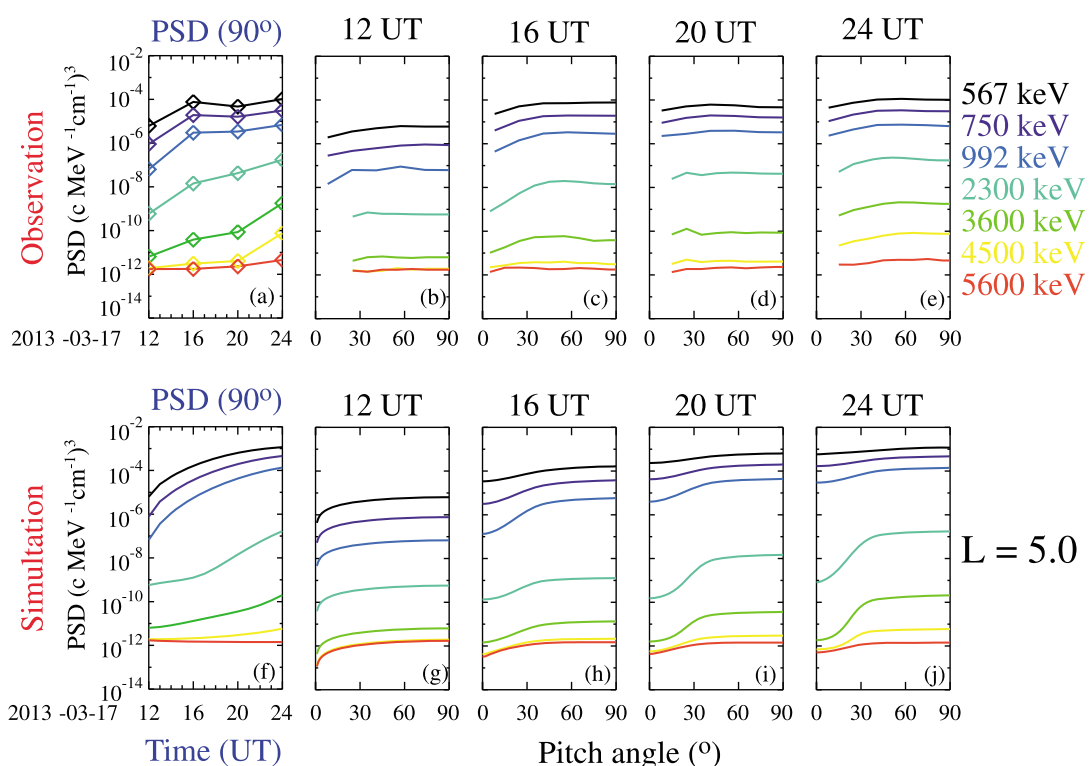

Pitch angle $\left({ }^{\circ}\right)$

Figure 7. The same as Figure 5 but at $L=5.0$.

We also performed similar simulations at different $L$-shells of 3.5 and 5.0 away from the PSD peak, as shown in Figures 6 and 7 respectively. At $L=3.5$ (Figure 6) although the simulated electron PSD captured the essential evolution of the observed PSD, the simulated values at energies above $\sim 2 \mathrm{MeV}$ are slightly lower than the observed PSD. This might be due to the absence of the inward radial diffusion process in our simulation, which could increase the PSD inside the location of the PSD peak. At $L=5.0$ (Figure 7), the observed electron pitch angle distribution after $\sim 20$ UT became very flat, different from the distribution at $L=3.5$ and 4.25 . This is probably related to the effect of outward radial diffusion, which is relatively stronger at higher $L$-shells caused by the gradient from the PSD peak $(L \sim 4.25)$ toward the higher $L$-shells, where losses caused by the magnetopause shadowing are more significant. The simulated electron PSD driven by chorus wave acceleration at $L=5.0$ is also generally lower than the observed values at energies above $3 \mathrm{MeV}$, which suggests that the outward radial diffusion may have contributed to increase the electron PSD. This is also consistent with the flattened radial electron PSD profile at $L^{*}>\sim 4.2$ after $\sim 20$ UT, as shown from Pass 7 to 12 in Figure 2. Note that the simulated electron PSD below $\sim 1 \mathrm{MeV}$ is generally larger than the observed values at the end of the simulation at all three different $L$-shells. This is probably due to the lack of other loss processes in the simulation, such as electron loss due to the open drift trajectory [Chen et al., 2003], pitch angle scattering by hiss in plumes, which is more effective for lower energies [Li et al., 2007; Summers et al., 2008; Ni et al., 2013], and radial diffusion process [e.g., Perry et al., 2005; Turner et al., 2012], as discussed above.

\section{Summary and Discussions}

In the present paper, using the global chorus wave intensity distribution inferred from low-altitude electron measurements, we quantify the role of chorus waves in energetic electron dynamics during the 17 March 2013 storm, when energetic electrons experienced very efficient acceleration within $\sim 12 \mathrm{~h}$. Our simulation results using 2-D quasi-linear diffusion theory show remarkable agreement with the observed electron PSD evolution near the PSD peak location at $L \sim 4.25$. Particularly, our simulation successfully reproduced the electron PSD changes in magnitude, timing, energy dependence, and the shape of the electron pitch angle distribution. At a lower $L$-shell of 3.5, our simulated electron PSD is slightly lower than the observed values, which might be related to our neglect of the inward radial diffusion process. At a higher L-shell of 5.0, our simulated electron PSD is also smaller than the observed value, which may be due to the lack of outward radial diffusion, which could increase the PSD at larger L-shells away from the PSD peak. Overall, our simulation driven by the interaction between chorus waves and energetic electrons is able to reproduce the essential features of the electron PSD evolution near the PSD peak location, which indicates that chorus waves play a dominant role in accelerating the seed electrons to highly relativistic energies during this geomagnetic storm. 
Although chorus waves are shown to play a key role in the electron PSD evolution, other processes need to be taken into account in order to fully understand the radiation belt electron dynamics. While our 2-D Fokker-Planck simulation was able to capture the essential features of the electron PSD evolution near the PSD peak location, the simulation results away from the peak location underestimated the actual electron PSD. This is probably because we did not include the radial diffusion process, which could modify the PSD profile away from the PSD peak location as discussed above. Furthermore, the observed PSD evolution is the result of a net competition of all acceleration and loss processes. In our simulation, we did not evaluate the loss processes during this geomagnetic storm, other than the loss caused by the chorus itself. The Van Allen Probes observed modestly strong $\mathrm{He}^{+}$band electromagnetic ion cyclotron (EMIC) waves at $\sim 3 R_{\mathrm{E}}$ on the nightside during the March 17 storm, but not at larger L-shells above 3.5 (not shown). The signature of electron pitch angle distribution driven by EMIC waves, such as shown in Li et al. [2007], was not clearly observed by the Van Allen Probes electron measurements. Therefore, loss processes driven by EMIC waves may not be significant in the heart of the outer radiation belt in this particular storm.

In this simulation, we also did not include the scattering loss caused by hiss in plumes. Although Van Allen Probes with the apogee located near the midnight sector and THEMIS with the apogee near 9 MLT did not directly observe a plume structure, the presence of a plume in the afternoon sector during this storm was captured from the GPS satellite observations [Foster et al., 2014]. Consequently, any hiss in the plumes will cause additional electron scattering particularly at larger pitch angles and lower energies (<1 MeV) [e.g., Li et al., 2007; Summers et al., 2008; Ni et al., 2013], which will need to be included to reproduce the observed electron pitch angle distribution.

In our 2-D simulation, we made several assumptions including chorus wave frequency spectrum, their latitudinal extension, wave normal distribution, and plasma density at certain MLT sectors (except for 00-04 MLT and 20-24 MLT where the density measurements from Van Allen Probes were available), since the in situ measurements on such parameters are limited. These parameters may also modify the simulation results, and the sensitivity of the PSD evolution dependent on the variation of these parameters is beyond the scope of this paper and is left for a future study.

Nevertheless, our simulation using the available in situ wave and particle measurements and the global chorus wave intensity inferred from the two-directional POES electron measurements clearly indicate that chorus-driven electron acceleration is a dominant process during this 17 March 2013 storm event. It is important to note that our conclusion on the dominance of chorus in the local acceleration of electrons to relativistic energies is different from the recent assertions of Shprits et al. [2013], who claim that a different process is responsible for highly relativistic electron acceleration during storms. This study, together with other previous studies simulating electron acceleration events [e.g., Horne et al., 2005a; Albert et al., 2009; Glauert et al., 2014; Shprits et al., 2009; Xiao et al., 2009; Su et al., 2010; Thorne et al., 2013; Tu et al., 2014; Turner et al., 2014], suggests that the chorus-driven electron acceleration is a robust and ubiquitous process in the Earth's inner magnetosphere and plays a crucial role in accelerating injected seed electrons with convective energies $(\sim 100 \mathrm{keV})$ to highly relativistic energies (several $\mathrm{MeV})$ in the outer radiation belt.

\section{References}

Agapitov, O., A. Artemyev, V. Krasnoselskikh, Y. V. Khotyaintsev, D. Mourenas, H. Breuillard, M. Balikhin, and G. Rolland (2013), Statistics of whistler-mode waves in the outer radiation belt: Cluster STAFF-SA measurements, J. Geophys. Res. Space Physics, 118, 3407-3420, doi:10.1002/jgra.50312.

Albert, J. M., N. P. Meredith, and R. B. Horne (2009), Three-dimensional diffusion simulation of outer radiation belt electrons during the 9 October 1990 magnetic storm, J. Geophys. Res., 114, A09214, doi:10.1029/2009JA014336.

Artemyev, A. V., O. V. Agapitov, D. Mourenas, V. Krasnoselskikh, and L. M. Zelenyi (2013), Storm-induced energization of radiation belt electrons: Effect of wave obliquity, Geophys. Res. Lett., 40, 4138-4143, doi:10.1002/grl.50837.

Baker, D. N., and S. G. Kanekal (2008), Solar cycle changes, geomagnetic variations, andenergetic particle properties in the inner magnetosphere, J. Atmos. Sol. Terr. Phys., 70, 195-206.

Baker, D. N., et al. (2012), The Relativistic Electron-Proton Telescope (REPT) instrument on board the Radiation Belt Storm Probes (RBSP) spacecraft: Characterization of Earth's radiation belt high-energy particle populations, Space Sci. Rev., 179, 337-381, doi:10.1007/s11214-012-9950-9.

Baker, D. N., et al. (2014), Gradual diffusion and punctuated phase space density enhancements of highly relativistic electrons: Van Allen Probes observations, Geophys. Res. Lett., 41, 1351-1358, doi:10.1002/2013GL058942.

Blake, J. B., et al. (2013), The Magnetic Electron lon Spectrometer (MagEIS) instruments aboard the Radiation Belt Storm Probes (RBSP) spacecraft, Space Sci. Rev., doi:10.1007/s11214-013-9991-8.

Bortnik, J., and R. M. Thorne (2007), The dual role of ELF/VLF chorus waves in the acceleration and precipitation of radiation belt electrons, J. Atmos. Sol. Terr. Phys., 69, 378-386. Nigel Meredith for their assistance in evaluating this paper. 
Chen, M. W., M. Schulz, G. Lu, and L. R. Lyons (2003), Quasi-steady drift paths in a model magnetosphere with AMIE electric field: Implications for ring current formation, J. Geophys. Res., 108(A5), 1180, doi:10.1029/2002JA009584.

Chen, Y., G. D. Reeves, and R. H. W. Friedel (2007), The energization of relativistic electrons in the outer Van Allen radiation belt, Nat. Phys., 3, doi:10.1038/nphys655.

Chen, Y., G. D. Reeves, R. H. W. Friedel, and G. S. Cunningham (2014), Global time-dependent chorus maps from low-Earth-orbit electron precipitation and Van Allen Probes data, Geophys. Res. Lett., 41, 755-761, doi:10.1002/2013GL059181.

Evans, D. S., and M. S. Greer (2004), Polar Orbiting Environmental Satellite Space Environment Monitor-2: Instrument descriptions and archive data documentation, NOAA Tech. Mem. 93, version 1.4, Space Weather Predict. Cent., Boulder, Colo.

Foster, J. C., P. J. Erickson, A. J. Coster, S. Thaller, J. Tao, J. R. Wygant, and J. W. Bonnell (2014), Storm time observations of plasmasphere erosion flux in the magnetosphere and ionosphere, Geophys. Res. Lett., 41, 762-768, doi:10.1002/2013GL059124.

Glauert, S. A., R. B. Horne, and N. P. Meredith (2014), Three-dimensional electron radiation belt simulations using the BAS Radiation Belt Model with new diffusion models for chorus, plasmaspheric hiss, and lightning-generated whistlers, J. Geophys. Res. Space Physics, 119, 268-289, doi:10.1002/2013JA019281.

Green, J. C. (2013), MEPED Telescope Data Processing Algorithm Theoretical Basis Document, Natl. Oceanic and Atmos. Admin. Space Environ. Cent., Boulder, Colo.

Green, J. C., and M. G. Kivelson (2004), Relativistic electrons in the outer radiation belt: Differentiating between acceleration mechanisms, J. Geophys. Res., 109, A03213, doi:10.1029/2003JA010153.

Horne, R. B., and R. M. Thorne (1998), Potential waves for relativistic electron scattering and stochastic acceleration during magnetic storms, Geophys. Res. Lett., 25(15), 3011-3014, doi:10.1029/98GL01002.

Horne, R. B., and R. M. Thorne (2003), Relativistic electron acceleration and precipitation during resonant interactions with whistler-mode chorus, Geophys. Res., Lett., 30(10), 1527, doi:10.1029/2003GL016973.

Horne, R. B., et al. (2005a), Wave acceleration of electrons in the Van Allen radiation belts, Nature, 437, 227-230, doi:10.1038/nature03939.

Horne, R. B., R. M. Thorne, S. A. Glauert, J. M. Albert, N. P. Meredith, and R. R. Anderson (2005b), Timescale for radiation belt electron acceleration by whistler mode chorus waves, J. Geophys. Res., 110, A03225, doi:10.1029/2004JA010811.

Iles, R. H. A., N. P. Meredith, A. N. Fazakerley, and R. B. Horne (2006), Phase space density analysis of the outer radiation belt energetic electron dynamics, J. Geophys. Res., 111, A03204, doi:10.1029/2005JA011206.

Kataoka, R., and Y. Miyoshi (2008), Magnetosphere inflation during the recovery phase of geomagnetic storms as an excellent magnetic confinement of killer electrons, Geophys. Res. Lett., 35, L06S09, doi:10.1029/2007GL031842.

Kennel, C. F., and H. E. Petschek (1966), Limit on stably trapped particle fluxes, J. Geophys. Res., 71(1), 1-28, doi:10.1029/JZ071i001p00001.

Kletzing, C. A., et al. (2013), The Electric and Magnetic Field Instrument Suite and Integrated Science (EMFISIS) on RBSP, Space Sci. Rev. doi:10.1007/s11214-013-9993-6.

Lam, M. M., R. B. Horne, N. P. Meredith, S. A. Glauert, T. Moffat-Griffin, and J. C. Green (2010), Origin of energetic electron precipitation $>30$ $\mathrm{keV}$ into the atmosphere, J. Geophys. Res., 115, A00F08, doi:10.1029/2009JA014619.

Li, W., Y. Y. Shprits, and R. M. Thorne (2007), Dynamic evolution of energetic outer zone electrons due to wave-particle interactions during storms, J. Geophys. Res., 112, A10220, doi:10.1029/2007JA012368.

Li, W., J. Bortnik, R. M. Thorne, and V. Angelopoulos (2011), Global distribution of wave amplitudes and wave normal angles of chorus waves using THEMIS wave observations, J. Geophys. Res., 116, A12205, doi:10.1029/2011JA017035.

Li, W., B. Ni, R. M. Thorne, J. Bortnik, J. C. Green, C. A. Kletzing, W. S. Kurth, and G. B. Hospodarsky (2013), Constructing the global distribution of chorus wave intensity using measurements of electrons by the POES satellites and waves by the Van Allen Probes, Geophys. Res. Lett., 40, 4526-4532, doi:10.1002/grl.50920.

Li, W., et al. (2014), Quantifying hiss-driven energetic electron precipitation: A detailed conjunction event analysis, Geophys. Res. Lett., 41, 1085-1092, doi:10.1002/2013GL059132.

Li, X., D. N. Baker, T. P. O'Brien, L. Xie, and Q. G. Zong (2006), Correlation between the inner edge of outer radiation belt electrons and the innermost plasmapause location, Geophys. Res. Lett., 33, L14107, doi:10.1029/2006GL026294.

Mauk, B. H., N. J. Fox, S. G. Kanekal, R. L. Kessel, D. G. Sibeck, and A. Ukhorskiy (2012), Science Objectives and Rationale for the Radiation Belt Storm Probes Mission, Space Sci. Rev., 1-15, doi:10.1007/s11214-012-9908-y.

McPherron, R. L., D. N. Baker, and N. U. Crooker (2009), Role of the Russell-McPherron effect in the acceleration of relativistic electrons, J. Atmos. Sol. Terr. Phys., 71, 1032-1044.

Meredith, N. P., R. B. Horne, A. Sicard-Piet, D. Boscher, K. H. Yearby, W. Li, and R. M. Thorne (2012), Global model of lower band and upper band chorus from multiple satellite observations, J. Geophys. Res., 117, A10225, doi:10.1029/2012JA017978.

Ni, B., R. M. Thorne, Y. Y. Shprits, and J. Bortnik (2008), Resonant scattering of plasma sheet electrons by whistler-mode chorus: Contribution to diffuse auroral precipitation, Geophys. Res. Lett., 35, L11106, doi:10.1029/2008GL034032.

Ni, B., R. M. Thorne, N. P. Meredith, R. B. Horne, and Y. Y. Shprits (2011), Resonant scattering of plasma sheet electrons leading to diffuse auroral precipitation: 2. Evaluation for whistler mode chorus waves, J. Geophys. Res., 116, A04219, doi:10.1029/2010JA016233.

Ni, B., J. Bortnik, R. M. Thorne, Q. Ma, and L. Chen (2013), Resonant scattering and resultant pitch angle evolution of relativistic electrons by plasmaspheric hiss, J. Geophys. Res. Space Physics, 118, 7740-7751, doi:10.1002/2013JA019260.

Ni, B., W. Li, R. M. Thorne, J. Bortnik, J. C. Green, C. A. Kletzing, W. S. Kurth, G. B. Hospodarsky, and M. de Soria-Santacruz Pich (2014), A nove technique to construct the global distribution of whistler-mode chorus wave intensity using low-altitude POES electron data, J. Geophys. Res. Space Physics, doi:10.1002/2014JA019935.

Perry, K. L., M. K. Hudson, and S. R. Elkington (2005), Incorporating spectral characteristics of Pc5 waves into three-dimensional radiation belt modeling and the diffusion of relativistic electrons, J. Geophys. Res., 110, A03215, doi:10.1029/2004JA010760.

Reeves, G. D., K. L. McAdams, R. H. W. Friedel, and T. P. O'Brien (2003), Acceleration and loss of relativistic electrons during geomagnetic storms, Geophys. Res. Lett., 30(10), 1529, doi:10.1029/2002GL016513.

Reeves, G., et al. (2013), Electron acceleration in the heart of the Van Allen radiation belts, Science, doi:10.1126/science.1237743.

Sheeley, B. W., M. B. Moldwin, H. K. Rassoul, and R. R. Anderson (2001), An empirical plasmasphere and trough density model: CRRES observations, J. Geophys. Res., 106(A11), 25,631-25,641, doi:10.1029/2000JA000286.

Shprits, Y. Y., and B. Ni (2009), Dependence of the quasi-linear scattering rates on the wave-normal distribution for chorus waves in the radiation belt, J. Geophys. Res., 114, A11205, doi:10.1029/2009JA014223.

Shprits, Y. Y. M., R. M. Thorne, R. Friedel, G. D. Reeves, J. Fennell, D. N. Baker, and S. G. Kanekal (2006), Outward radial diffusion driven by losses at magnetopause, J. Geophys. Res., 111, A11214, doi:10.1029/2006JA011657.

Shprits, Y. Y., D. Subbotin, and B. Ni (2009), Evolution of electron fluxes in the outer radiation belt computed with the VERB code, J. Geophys. Res., 114, A11209, doi:10.1029/2008JA013784. 
Shprits, Y. Y., D. Subbotin, A. Drozdov, M. E. Usanova, A. Kellerman, K. Orlova, D. N. Baker, D. L. Turner, and K.-C. Kim (2013), Unusual stable trapping of the ultrarelativistic electrons in the Van Allen radiation belts, Nat. Phys., doi:10.1038/nphys 2760.

Shue, J. H., et al. (1998), Magnetopause location under extreme solar wind conditions, J. Geophys. Res., 103(A8), 17, 691-17,700, doi:10.1029/98JA01103.

Spence, H. E., et al. (2013), Science Goals and Overview of the Energetic Particle, Composition, and Thermal Plasma (ECT) Suite on NASA's Radiation Belt Storm Probes (RBSP) Mission, Space Sci. Rev., doi:10.1007/s11214-013-0007-5.

Su, Z., F. Xiao, H. Zheng, and S. Wang (2010), STEERB: A three-dimensional code for storm-time evolution of electron radiation belt, J. Geophys. Res., 115, A09208, doi:10.1029/2009JA015210.

Summers, D., C. Ma, N. P. Meredith, R. B. Horne, R. M. Thorne, D. Heynderickx, and R. R. Anderson (2002), Model of the energization of outer-zone electrons by whistler-mode chorus during the October 9, 1990 geomagnetic storm, Geophys. Res. Lett., 29(24), 2174, doi:10.1029/2002GL016039.

Summers, D., B. Ni, N. P. Meredith, R. B. Horne, R. M. Thorne, M. B. Moldwin, and R. R. Anderson (2008), Electron scattering by whistler-mode ELF hiss in plasmaspheric plumes, J. Geophys. Res., 113, A04219, doi:10.1029/2007JA012678.

Thorne, R. M. (2010), Radiation belt dynamics: The importance of wave-particle interactions, Geophys. Res. Lett., 37, L22107, doi:10.1029/2010GL044990.

Thorne, R. M., B. Ni, X. Tao, R. B. Horne, and N. P. Meredith (2010), Scattering by chorus waves as the dominant cause of diffuse auroral precipitation, Nature, 467, 943-946, doi:10.1038/nature09467.

Thorne, R. M., et al. (2013), Rapid local acceleration of relativistic radiation belt electrons by magnetospheric chorus, Nature, 504, 411-414, doi:10.1038/nature12889.

Tsyganenko, N. A., and M. I. Sitnov (2005), Modeling the dynamics of the inner magnetosphere during strong geomagnetic storms, J. Geophys. Res., 110, A03208, doi:10.1029/2004JA010798.

Tu, W., G. S. Cunningham, Y. Chen, S. K. Morley, G. D. Reeves, J. B. Blake, D. N. Baker, and H. Spence (2014), Event-specific chorus wave and electron seed population models in DREAM3D using the Van Allen Probes, Geophys. Res. Lett., 41, 1359-1366, doi:10.1002/2013GL058819.

Turner, D. L., Y. Shprits, M. Hartinger, and V. Angelopoulos (2012), Explaining sudden losses of outer radiation belt electrons during geomagnetic storms, Nat. Phys., 8, 208-212, doi:10.1038/NPHYS2185.

Turner, D. L., V. Angelopoulos, W. Li, M. D. Hartinger, M. Usanova, I. R. Mann, J. Bortnik, and Y. Shprits (2013), On the storm-time evolution of relativistic electron phase space density in Earth's outer radiation belt, J. Geophys. Res. Space Physics, 118, 2196-2212, doi:10.1002/jgra.50151.

Turner, D. L., et al. (2014), Competing source and loss mechanism due to wave-particle interactions in Earth's outer radiation belt during the 30 Sep.-03 Oct. 2012 geomagnetic storm, J. Geophys. Res. Space Physics, 119, 1960-1979, doi:10.1002/2014JA019770.

Ukhorskiy, A. Y., M. I. Sitnov, K. Takahashi, and B. J. Anderson (2009), Radial transport of radiation belt electrons due to stormtime Pc5 waves, Ann. Geophys., 27, 2173-2181.

Xiao, F., Z. Su, H. Zheng, and S. Wang (2009), Modeling of outer radiation belt electrons by multidimensional diffusion process, J. Geophys. Res., 114, A03201, doi:10.1029/2008JA013580. 